\title{
Construction of Medical Dispute Prevention Mechanism
}

\author{
Longfei Jiang* \\ School of Humanity and Law, Wuhan University of \\ Technology \\ Wuhan, China \\ 1515779581@qq.com
}

\author{
Jingying Xiang \\ School of Humanity and Law, Wuhan University of \\ Technology \\ Wuhan, China \\ 765964123@qq.com
}

\begin{abstract}
The increasing medical dissension makes the unhealthy development of medical and health service and affects the harmonious and stable social life of our country. Therefore, it has become an important problem to be solved in the social life on how to resolve medical disputes, cure "medical trouble" [1] and set up a good preventive mechanism of medical disputes; the urgent affair is to find the primary cause of "medical trouble" and prevent the birth of medical disputes from the origin. A sound medical dispute prevention mechanism should be formulated through the synchronized sharing of medical records and the determination of scope of validity of patient's informed consent; Based on the current situation of medical disputes, this paper finds out the problems existing in the treatment mechanism of medical disputes in China, and then analyzes the basic causes of medical disputes. It also demonstrates the defects in medical disputes and the unreasonable internal structure of medical institutions; this paper expounds the standard of adequacy of physician's explanation of obligation, and perfects the existing medical dispute prevention mechanism according to the sharing system of medical record and informed consent standard.
\end{abstract}

Keywords-Medical disputes; Medical trouble; Hysteretic law; Synchronous sharing; Informed consent; Prevention mechanism;

\section{INTRODUCTION}

At present, China is in the high-incidence season of medical malpractice [2]. As for the hospital self-protection and patient right-safeguard, an important topic is how to build the preventive medical dispute resolution mechanism and nip the medical negligence in the bud because the topic is very realistic significance to save the limited medical resources and keep the normal medical treatment order. Meanwhile, what's the source of the increasingly strained doctor-patient relations. The author has analyzed the various medical disputes and complaints in recent years to discuss the medical dispute causes and how to eradicate the medical dispute according to the internship experience.

\section{THE STATUS AND CAUSE OF MEDICAL DISPUTES}

\section{A. Current situation of medical dispute}

Since Some Provisions on Civil Procedure Evidences and Medical Malpractice Management Regulation were published in 2002, our medical dispute has increased by 26.41\%, $83.31 \%$ of which is compromised by hospital and patients, and $10.48 \%$ dispute is solved in the legal actions [3]. In recent years, our medical dispute quantity is increased year by year. Specifically, there are nearly 1 million disputes throughout the country every year, and averagely every hospital is faced with 40 disputes every year. As for the Class III Grade I hospitals, there are 100 claim cases every year at least, 20-30 cases are solved by People's Court; however, the compensation amount is increased year by year. In 2013, the national medical institutions suffered from 70,000 medical disputes. What's worse, the following cases were published on the media: Case Concerning False Transfusion Death of Hospital, Infant Suspected "Roasted to Death" Event, A Man of Wenling, Zhejiang Kills Doctor with a Knife, and so on. Every case has exerted the strong social response and worsened the strained doctor-patient relations.

\section{B. The cause of medical disputes}

\section{a) Unbalanced medical and health resources}

The medical and health resource allocation refers to the distribution and flow of medical and health resources in the medical and health industry, including health and resource allocation, namely, incremental allocation and inventory adjustment, which are also known as "initial allocation" and "readjustment" [4]. As an important constituent part and relatively active part of medical and health resources, the unbalanced distribution and unfair allocation are deemed as the most remarkable problems during the medical and health human resources allocation in China. Particularly, the urbanrural imbalance, as a prominent problem in the fairness and efficiency research about medical \& health resource allocation and health system, has serious impacts on the fairness and efficiency objectives of resource allocation [5]. First of all, there are obvious unbalance and unfairness between cities and rural areas in the aspect of medical and health human resources, such as medical practitioners and health workers distribution; Secondly, the medical and health human resources allocation also shows the structural disequilibrium caused by urban-rural unbalance in the aspect of high-quality resources and worker quality, such as the high-quality medical and health workers always work in the large-scale medical institutions in cities, much higher than the medical staff who work in the rural and grass-root medical institutions, as a result the medical 
technology and service ability of medical staff are restricted to lower the medical and health service quality in rural areas [6]. This is an urgent problem which needs to be solved by Chinese health system. How to allocate and utilize the medical and health human resources fairly and rationally is a problem which concerns the health level and resource utilization efficiency of residents in a country or region.

\section{b) The "high requirement" of the patients}

The blocked complaint and right-safeguard channel is deemed as the direct reason which can affect the doctor-patient relations. Although China has enforced the new Medical Malpractice Management Regulation a few years ago, it is still difficult for patients to protect their profits through the normal channel after medical malpractice because of the high cost of profit protection. For example, the accident identification expenses are always several thousand Yuan. In such case, the self-protection is preferred, namely, the medical dispute is solved by the illegal or violent means, such as cry, scream and hit to claim for compensations.

The patients' demands for hospital are getting higher and higher, including "diversified" medical service. In consideration of the high specialization of medical field and the large gap between hospital and patients in the aspect of cognitive level about medical knowledge, the patients are unable to understand the non-detectable technology, incurable disease or the unavoidable adverse effects caused by medical events regardless of its visibility. When there is any deviation between patients' expectation and treatment effects of hospital, the patients are likely to feel dissatisfied, what's worse, some reasonless patients or family members adopt the extreme means to expand the dispute.

\section{c) The hysteretic law}

Chinese laws and regulations about medical dispute resolution are relatively imperfect; as a result, the concepts are vaguely separated from medical system. The Medical Malpractice Management Regulation is not applicable to the judicial identification and civil compensation caused by medical disputes because it is an administrative regulation; According to "shifting burden of proof" as specified in Some Provisions on Civil Procedure Evidences enacted by Supreme People's Court, the medical institutions are always passive during medical dispute resolution. For patients, it is difficult to put to the proof during medical dispute; Therefore, it can be said that the Supreme People's Court has given the overweight responsibility to medical institutions according to this judicial interpretation, as a result, the compensation responsibility of medical institutions is also enlarged; At present, the burden of proof implementation in the medical malpractice is still disputable.

\section{THE CONSTRUCTION OF MEDICAL PREVENTION MECHANISM}

At present, the frequent occurrence of medical disputes has become the main factor affecting the normal working order of medical and health units, which not only damages the image of the hospital and affects the social and economic benefits of the unit, but also hinders the medical innovation and the development of medical science, and harms the interests of the masses. It has even threatened the safety of the vast number of medical personnel. The medical personnel will not take $99 \%$ of the risk to seek for $1 \%$ hope in the rescue of patients. In recent years, in order to prevent and deal with medical disputes, we have actively explored the practice and found out a set of methods and measures to prevent medical disputes; Through the medical record sharing and informed consent, we should pay close attention to the quality management, perfect the management system, strengthen the communication between doctors and patients and improve the medical environment to fundamentally eradicate the internal factors of the medical

\section{A. Synchronization sharing mechanism of medical record information}

\section{a) Specification of medical records}

The medical records related to the disease diagnosis and treatment process of patients shall be objective, authentic and scientific, otherwise, its required value will be lost. Therefore, the medical records shall satisfy the specific requirements. First of all, the medical records shall be prompt; the outpatient medical records will start with the contact and exchange between doctor and patients until the diagnosis and treatment are over. The admission records shall cover the medical activities in the hospital admission date. Secondly, the medical records shall be specific; Contents include: Time, situation at that time, diagnosis name, diagnosis and treatment process, disease evolution and other contents. If the patient is unstable, any new change shall be recorded faithfully. Thirdly, the medical records shall be objective; the medical records shall be written on the basis of the objective data and indicators during the signs and symptoms inspection. Any speciosity and assumed reasoning will be prohibited. The analysis or discussion of doctors shall be also recorded faithfully for further inquiry.

\section{b) Malpractice of unilateral medical record keeping}

The writing and storage of medical records, as well as the reprography right of patients are stipulated in Item 1, Clause 8 and Item 1, Clause 10 of Medical Malpractice Management Regulation. However, the reprography time of patients is unnoticed in the foregoing regulation. In practice, the patients are unlikely to get and print their medical records in the process of medical treatment. When the medical dispute occurs, the hospital will be reluctant to hand over the medical records copies to patients only when patients raise the claim. Due to the unilateral generation, unilateral management of medical records and unnoticed reprography requirements, the medical staff has the opportunity to counterfeit and falsify the medical records. According to the statistics, the success rate of medical records counterfeit and falsification is larger than $80 \%$. During the lawsuit, the counterfeited medical records account for $30 \%$ in the total number at least [7]. However, the patients will be suspicious of the medical records authenticity under the extremely distrustful doctor-patient relations and such management system even if the medical records are not falsified. Given this, the author advises the hospital to divide 
the medical diagnosis and treatment into courses or time periods, and copy the medical records for patients actively, in such case, the patients will know their illness conditions and understand the doctors' efforts and painstaking in the process of diagnosis and treatment; On the other hand, it is feasible to establish the restriction mechanism about medical records and storage, therefore, the patients will have trust in the medical behaviors of doctors to avoid the medical disputes.

\section{c) Synchronous sharing function of medical records information}

According to the related laws and regulations, the medical records shall be directly written and produced by attending physicians, and the hospital is responsible for collecting and storing the medical records, so the scientific research and legal value are available. First of all, the medical records shall have evidence value. In order to put to the proof to clear up the medical malpractice, the medical institutions shall make it on the basis of the authentic medical records. If both parties can share the medical records in the process of medical activities, it is good for keeping the authenticity of medical records, strengthening the patients' trust in hospital and enhancing the proving capacity of evidence. In such case, they will take the legal responsibility according to laws to avoid the disordered development of contradictions. Secondly, it can make up the insufficient information disadvantages for patients in the process of diagnosis and treatment. Therefore, the rationality understanding of diagnosis and treatment behaviors and patients' status promotion are deemed as the comprehension and respect for patients.

\section{B. Informed and consent system}

\section{a) Obligations of doctor to disclose}

The disclosure obligations of physicians are stipulated in Clause 26 of Medical Practitioner Law of People's Republic of China; The Clause 33 of Medical Institutions Management Code and Clause 11 of Medical Malpractice Management Regulation have prescribed the informed consent right of patients, family members or stakeholders for surgery, special inspection or treatment. The reason is that the medical behavior is a kind of dangerous behavior which is likely to encroach on the patients' life and body health, and the medical malpractice will make inroads on the life right and health right of patients, especially the invasive treatment or inspection is liable to bring trauma and corresponding side effect to the patients at different degrees. Therefore, the medical staff shall fully explain the treatment process, curative effect, the probable complications, as well as the success or failure rate of treatment scheme, and get the understanding of patients or family members. This is the premise of legitimate medical behaviors.

\section{b) The judgment of the adequacy standard}

From the perspective of informed consent development history, the explanation adequacy of doctors shall be judged according to two standards, namely, patients' standard, which should the patients know during the suggested treatment behaviors, in other words, confirm whether the doctors have informed patients of the substantial importance when they carry out the "informed" medical behaviors. However, the patients serve as the judgment subject when the substantial importance of this information is judged. In consideration of the limited medical knowledge of patients and mutual distrust between hospital and patients, the foregoing standard shall not be used because such subjective standard is liable to increase the responsibility burden of doctors. Given this, the author agrees with the second standard: Rational doctor standard, namely, the professional standard. According to this standard, the doctors shall abide by the following explanation standards when the doctors' explanation adequacy is confirmed, namely, what kind of mode and information that the normal and rational doctors explain to patients in the same or similar conditions on the basis of the operation program and convention in the medical industry [8]. Its rationality is that the layman is unable to evaluate the medical decision adaptability related to the explanation obligations fulfillment in the reasonable way when the medical behaviors are professional. Therefore, the doctors' explanation about diagnosis and treatment behaviors will be deemed to be adequate as long as the explanation has satisfied the professional standards provided by a rational doctor.

\section{c) The effectiveness of informed consent}

In nature, the informed consent opinion signed by patients is a supporting document. The efficacy of this supporting document is embodied in three aspects: Firstly, probative effect in the procedural law, namely, when patients have affixed signature, it means that the medical institution and medical staff have informed the patients of medical behaviors and got approval from patients. This is the consent in form; Secondly, probative effect in substantive law, namely, the medical institutions and medical staff are truly proved to fulfill the truthful inform obligations to patients and family members, meanwhile, it also means that the patients have known the deserved damage and probable accidents caused by the medical behaviors [9]. Therefore, the form and substantive efficacy of informed consent can realize the medical behaviors' function to hinder the illegality. Thirdly, the informed consent has also shown the doctors' respect for patients' personal dignity and right to know. As a basic clinical ethical principle, the informed consent has a real effect on the implementation of self-decision right of patients and the perfection of doctorpatient trust relations.

\section{CONCLUSION}

By adhering to the principle of prevention and treatment of medical disputes of "Uphold both the prevention and control, preventing the main", we should pay close attention to the medical quality management and controlling, prevent and reduce the occurrence of medical disputes, resolve and deal with medical disputes in time, prevent the aggravation and enlargement of medical disputes, improve the quality of medical The treatment of medical disputes is becoming more and more mature, and the adverse effects of medical disputes in society are declining. So that the hospital in the development and construction of the broad masses of support, and can better serve patients, so as to truly establish a harmonious relationship between doctors and patients. 


\section{REFERENCES}

[1] Lan Yingchun, Wang Min, Wang Deguo. Review of "Medical Dispute Profiteer" Downtown, Article No.: 1001-8565(2008)06-0064-02 (In Chinese)

[2] Zuo Guoqing, Liao Yu. Porter Competitive Strategy and Hospital's SWOT Analysis after WTO [J]. Chongqing medicine, 2003,32(10): 1 461-1463. (In Chinese)

[3] Li Guoguang: Supreme People's Court's Understanding and Applicability to <Some Provisions on Civil Procedure Evidences>, China Legal Publishing House, 2002 Version. (In Chinese)

[4] Ren Ran, Difference and Development Thinking About Urban-Rural Health Resources Allocation [J]. China Health Human Resources, 2014, 6:16-17. (In Chinese)
[5] Zhu Lanfang, Existing Problems and Suggestions on the Financial Management of County-level Health System [J]. Journal of Clinical Medical Literature (Electronic Version), 2014, (1) (In Chinese)

[6] Xiao Lv. Brief Analysis of Measures to Strengthen the Financial Management Works in the Medical Health Field [J]. Money China, 2014 (27). (In Chinese)

[7] Lin Cunzhu. Medical Malpractice Lawsuit [M]. Beijing: People's Publishing House, 2006.214. (In Chinese)

[8] Hu Yongqing. Doctors' Disclosure Obligation Composition in the Informed Consent Theory [J]. Science of Law, 2005,(1): 91- 98. (In Chinese)

[9] [9] Chen Yiman. On Thinking of Medical Informed Consent in Law [J]. Medicine and Philosophy.(In Chinese) 